TITLE:

\title{
A spectrum-driven damage identification by minimum constitutive relation error and sparse regularization
}

\section{$\operatorname{AUTHOR}(S):$}

Guo, Jia; Jiao, Jian; Fujita, Kohei; Takewaki, Izuru

\section{CITATION:}

Guo, Jia ...[et al]. A spectrum-driven damage identification by minimum constitutive relation error and sparse regularization. Mechanical Systems and Signal Processing 2020, 136: 106496.

\section{ISSUE DATE:}

2020-02

URL:

http://hdl.handle.net/2433/245025

\section{RIGHT:}

(c) 2020. This manuscript version is made available under the CC-BY-NC-ND 4.0 license

http://creativecommons.org/licenses/by-nc-nd/4.0/.; The full-text file will be made open to the public on 1 February 2022 in accordance with publisher's 'Terms and Conditions for Self-Archiving'.; This is not the published version. Please cite only the published version.; この論文は出版社版でありません。引用の際には出版社版をご確認ご利用ください。 


\author{
Jia Guo*, ${ }^{*}$, Jian Jiao ${ }^{1}$, Kohei Fujita ${ }^{1}$, and Izuru Takewaki ${ }^{1}$ \\ ${ }^{1}$ Department of Architecture and Architectural Engineering, Kyoto University, Kyoto, \\ Japan
}

\begin{abstract}
This paper proposes a novel model-based damage identification strategy based on minimum constitutive relation error and sparse regularization using the power spectrum density data. Firstly, the stationary random vibration problem is transformed into a series of harmonic vibrations by the pseudo excitation method and the error in constitutive relation is established by the admissible stress field and admissible displacement field. A much more general and simpler strategy so as to build the admissible stress field is addressed by requiring only an extra decomposition of the stiffness matrix. Then, the sparse regularization is added to the original constitutive relation error objective function to circumvent the ill-posedness of the inverse problem. Finally, the solution of this nonlinear optimization problem is solved by the alternating minimization method. The proposed method has the advantage that only measurement power spectrum density data from few limited sensors are needed in the inverse analysis. Numerical and experimental results show the effectiveness and robustness of this approach.
\end{abstract}

Keywords: damage identification, constitutive relation error, sparse regularization, power spectrum density, pseudo excitation method

\title{
1 Introduction
}

Methods for damage identification are undergoing increased attention and development in the last decades [1-3]. Finite element model-based methods treat damage identification as a constrained nonlinear optimization problem in which the discrepancy between the test data and

\footnotetext{
${ }^{*}$ Correspondence to: Jia Guo, Email: kaku.ka.25x@st.kyoto-u.ac.jp
} 
the predictions from the numerical model is minimized. Many researches have shown that the finite element model-based methods are able to identify the damage location and severity simultaneously with suitable measurement data $[4,5]$. Three categories of objective functions can be used for damage identifications: the input residuals, the output residuals and the residual named the 'Constitutive relation error(CRE)'. The CRE, an energy inner product of the residual of the constitutive equation connecting the admissible stress field and admissible displacement field, provides a measure of quality of the updated model using the constitutive relation. The minimum constitutive relation error (min-CRE) approach is a priori suited for the identification of all generalized standard materials [6]. It was initially proposed for the identification of elastic properties [7] and now is being widely adjusted into nonlinear constitutive models, i.e., identification of heterogeneous elastoplastic properties [8] and strain energy densities [9]. The ability to include all available experimental information about displacements and stresses to define the basic identification problem allows this approach to deal with missing/less reliable boundary conditions efficiently [10]. A comparison between the min-CRE approach and the input/output residual method was conducted in [11] and the results showed that the min-CRE approach was more robust in the numerical examples where realistic perturbations were added and it could also give the same results as the input/output residual method even with less boundary information. Although the min-CRE approach accommodates full-field displacement measurements in a very natural way [12], obtaining full-field measurements for large-scale civil engineering structures is still a challenging issue. As a result, it is highly desirable to develop the min-CRE approach that could robustly address the ill-posed problem caused by the insufficient amount of measurement sensors during the identification process. The references [13] and [14] offered a new perspective on the problem by incorporating sparse regularization into the min-CRE approach to solve the ill-posed inverse problem due to the incomplete modal data and limited frequency response function data, respectively. It was shown that the sparse regularization could be added to the original optimization problem with little computational efforts and improve the effectiveness and robustness of the min-CRE approach under a relative high level of measurement noise.

This paper is based on the work of [14] and extends the sparse regularized min-CRE approach to spectrum-driven problems. It is well known that long-span structures or high-rise buildings can be excited well by non-deterministic random natural disturbances (seismic, wind, road irregularities, etc.) in their operating environment, making it desirable to use the corresponding random vibration responses as measurement data for damage identification directly. A description of the input excitation and output response in terms of power spectral density (PSD) 
is commonly used for random vibrations [15]. Compared to the usage of the modal data [13] and the frequency response function data [14], the advantage of using PSD data from a continuous frequency range without reducing the damage identification process to limited number of resonant frequencies or near resonance frequencies was observed, which could also be found in other research [16]. Inspired by the work in [17], the pseudo excitation method (PEM) $[18,19]$ which transforms a random vibration analysis into a series of harmonic response analysis is introduced, making the damage identification solvable under the random excitations.

Furthermore, a difficulty lies in how to obtain the admissible stress field satisfying the equilibrium constraints when building the CRE. In [14], the force finite element is directly involved to establish the stress field. This procedure is efficient and convenient with respect to the beamtype elements but may lead to solving sophistication for other types of elements. To avoid the recourse to stress/force finite element, this paper gives an alternate and simplified procedure for stress field building, requiring only an extra decomposition of the stiffness matrix under consideration. During this decomposition, damage parameters are physically extracted from the stiffness matrix and the admissible stress field is directly and simply built based on the equilibrium equation and the pseudoinverse of the generic matrix decomposed from the stiffness matrix. It should be noted that the procedure of stiffness matrix decomposition is only conducted at the initial stage in the proposed method, rather than at each iterative step, making this strategy efficient in its application.

The remainder of this paper is organized as follows. The basic idea of PEM for forward problem is reviewed in Section 2.1 and the min-CRE approach for inverse problems is briefly introduced in Section 2.2. The main solving process for this identification problem in Section 3 is threefold. Firstly, the stress field building strategy is illustrated in Section 3.1. Secondly, the sparse regularization is added to the original CRE function in Section 3.2 so as to improve the accuracy and robustness of the proposed approach when measured data are incomplete and the damage identification problem becomes ill-posed. Large-scale civil engineering structures contain a great number of elements whereas damage only occurs at limited members, which is appropriately implicated in the sparse regularization. Finally, in Section 3.3, the efficient alternating minimization (AM) method is applied to get the solution of this nonlinear optimization problem. The AM method is an iterative procedure for minimum solution of the separately convex objective function and hence no sensitivity analysis is involved during the solution process. In principle, the AM method tries to get the solution iteratively by alternating minimization of the objective function over every separated variable in each iteration. Numerical tests and 
experimental verification are performed in Section 4 and Section 5 separately. Final conclusions are drawn in Section 6.

\section{Identification problem to be solved}

\subsection{Pseudo excitation method (PEM) for forward problem}

Consider a general finite element model of a linear elastic time-invariant structure subjected to a single stationary random excitation $x_{0}(t)$ whose power spectral density $S_{0}(\omega)$ has been specified. The equation of motion can be written as

$$
\mathbf{M} \ddot{\boldsymbol{u}}+\mathbf{C} \dot{\boldsymbol{u}}+\mathbf{K} \boldsymbol{u}=\boldsymbol{a} x_{0}(t)
$$

where $\mathbf{M}, \mathbf{K}$ and $\mathbf{C}$ are the system mass, stiffness and damping matrices, respectively. $\mathbf{C}$ is assumed to be a Rayleigh damping herein and $\mathbf{C}=\alpha \mathbf{M}+\beta \mathbf{K}(\alpha$ and $\beta$ are two constants determined by two modal frequencies). $\boldsymbol{a}$ is a given constant vector. To solve this problem, let $x_{0}(t)=\sqrt{S_{0}(\omega)} e^{i \omega t}$, then the above equation of motion is reduced into the following harmonic equation:

$$
\mathbf{M} \ddot{\boldsymbol{u}}+\mathbf{C} \dot{\boldsymbol{u}}+\mathbf{K} \boldsymbol{u}=\boldsymbol{a} \sqrt{S_{0}(\omega)} e^{i \omega t}
$$

where $\sqrt{S_{0}(\omega)} e^{i \omega t}$ is the so called 'pseudo harmonic excitation' in the PEM and the corresponding pseudo harmonic response would be

$$
\boldsymbol{u}=\mathbf{H} \boldsymbol{a} \sqrt{S_{0}(\omega)} e^{i \omega t}
$$

where $\mathbf{H}$ is the frequency response function.

Note that the above equations only consider the cases with stationary random excitation due to the convenience of computation and the brevity of expression. The non-stationary pseudo excitation can be simply expressed by the stationary pseudo excitation multiplied by an envelope function $g(t)[20]$ (or in a more general way the frequency-time modulating function $A(t, \omega)$ [21]). Hence the identification method for structures under non-stationary random excitations is a natural extension of the present method for stationary random excitations. However, except for some special cases with simple envelope function $g(t)$, the pseudo responses $\boldsymbol{u}$ in Eq. (3) can only be calculated by means of the Duhamel integration formula or other numerical integration methods instead of directly being solved within the frequency domain as proposed in this article, which leads the overall identification process to high computational efforts. Nevertheless, for most of the earthquake ground motions, the non-stationary effects can be no trivial and thus the corresponding solving process merits attention. 


\section{$2.2 \quad$ Min-CRE approach for inverse problem}

For inverse identification problems, quite often the mass matrix $\mathbf{M}$ is considered to be accurate and only the stiffness matrix $\mathbf{K}$ needs to be modified. The basic idea in the damage parameter identification herein is to express the stiffness $\mathbf{K}$ in the form of the following decomposition

$$
\mathbf{K}(\boldsymbol{\theta})=\mathbf{L D}(\boldsymbol{\theta}) \mathbf{R}
$$

where $\mathbf{D}(\boldsymbol{\theta})$ is a diagonal matrix with the following expression,

$$
\mathbf{D}(\boldsymbol{\theta})=\left(\begin{array}{cccc}
\theta_{1} \mathbf{D}_{1} & \mathbf{0} & \cdots & \mathbf{0} \\
\mathbf{0} & \theta_{2} \mathbf{D}_{2} & \cdots & \mathbf{0} \\
\vdots & \vdots & \ddots & \vdots \\
\mathbf{0} & \mathbf{0} & \cdots & \theta_{m} \mathbf{D}_{m}
\end{array}\right)
$$

$\mathbf{D}_{e}$ is obtained from the coefficient matrix of the elastic modulus in elasticity for element $e$, $e=1, \cdots, m$ and $\boldsymbol{\theta}$ denotes the damage parameter, with $\theta_{e}=1$ meaning no damage of the eth element. $\mathbf{D}(\boldsymbol{\theta})$ is of size $r=\sum_{e=1}^{m} r_{e}, r_{e}=\operatorname{rank}\left(\mathbf{D}_{e}\right) . \mathbf{L}$ and $\mathbf{R}$ are derived from the strain-displacement matrix as well as numerical integration. The decomposition in Eq. (4) is not unique. Especially, there is

$$
\mathbf{K}(\boldsymbol{\theta}) \boldsymbol{u}=\mathbf{L} \boldsymbol{\sigma} .
$$

and thus the constitutive relation can be formulated in a discrete form as

$$
\boldsymbol{\sigma}=\mathbf{D}(\boldsymbol{\theta}) \mathbf{R} u
$$

where $\boldsymbol{\sigma}$ is a global column matrix assembled by each elemental stress. Different types of elements correspond to different decomposition. Appendix A.1 provides further insight into the proposed decomposition, detailed derivation of Eq. (6) as well as specific expressions of D, $\mathbf{L}$ and $\mathbf{R}$ for $1 \mathrm{D}$ spring element and 2D Euler-Bernoulli beam element used in the following sections. Moreover, readers are referred to literature $[22,23]$ for the similar idea of stiffness matrix decomposition for damage identification problem and literature [24] for the idea of the stiffness-related parameterization scheme.

For the sake of simplicity, we will work in the frequency domain and employ the same notation later for the associated amplitudes and the quantities. To define the constitutive relation error (CRE), consider an admissible pair $(\tilde{\boldsymbol{u}}, \tilde{\boldsymbol{\sigma}})$ which satisfies the kinematic constrains $(\tilde{\boldsymbol{u}} \in \mathcal{U})$ and the equilibrium equations $(\tilde{\boldsymbol{\sigma}} \in \Im)$, respectively. As already known, if the constitutive relation in Eq. (7) is additionally satisfied with an admissible damage parameter $\boldsymbol{\theta} \in \mathcal{A} \subseteq \mathbb{R}^{m+}$, the 
above admissible solution trio $(\tilde{\boldsymbol{u}}, \tilde{\boldsymbol{\sigma}}, \boldsymbol{\theta})$ would be the exact solution $\left(\tilde{\boldsymbol{u}}_{\mathrm{ex}}, \tilde{\boldsymbol{\sigma}}_{\mathrm{ex}}, \boldsymbol{\theta}_{\mathrm{ex}}\right)$. As a result, the CRE for a given frequency range $\left[\omega_{\min }, \omega_{\max }\right]$ is introduced as

$$
\begin{aligned}
e_{\mathrm{CRE}}(\tilde{\boldsymbol{u}}, \tilde{\boldsymbol{\sigma}}, \boldsymbol{\theta}) & =\frac{1}{2} \int_{\omega_{\min }}^{\omega_{\max }}(\mathbf{D}(\boldsymbol{\theta}) \mathbf{R} \tilde{\boldsymbol{u}}-\tilde{\boldsymbol{\sigma}})^{T} \mathbf{D}(\boldsymbol{\theta})^{-1}(\mathbf{D}(\boldsymbol{\theta}) \mathbf{R} \tilde{\boldsymbol{u}}-\tilde{\boldsymbol{\sigma}}) z(\omega) d \omega \\
& =\frac{1}{2} \int_{\omega_{\min }}^{\omega_{\max }}\left\|\mathbf{D}(\boldsymbol{\theta})^{-\frac{1}{2}}(\mathbf{D}(\boldsymbol{\theta}) \mathbf{R} \tilde{\boldsymbol{u}}-\tilde{\boldsymbol{\sigma}})\right\|^{2} z(\omega) d \omega
\end{aligned}
$$

where $z(\omega)$ is the frequency weighting factor which satisfies

$$
\int_{\omega_{\min }}^{\omega_{\max }} z(\omega) d \omega=1, z(\omega) \geq 0 .
$$

Eq. (8) is known as the CRE objective function for inverse identification problems [25, 26]. The value of $z(\omega)$ represents the relative reliability of the measurements at different frequencies and in this research, $z(\omega)$ is set as a constant equal to $1 /\left(\omega_{\max }-\omega_{\min }\right)$, which is generally used in other publications $[25,26]$.

Based on the equations above, the damage identification problem can be solved by the following program $\left(\mathrm{P}_{0}\right)$

$$
\begin{array}{ll}
\left(\mathrm{P}_{0}\right) & (\tilde{\boldsymbol{u}}, \tilde{\boldsymbol{\sigma}}, \boldsymbol{\theta})=\arg \min e_{\mathrm{CRE}}(\tilde{\boldsymbol{u}}, \tilde{\boldsymbol{\sigma}}, \boldsymbol{\theta}) \\
& \text { subject to } \tilde{\boldsymbol{u}} \in \mathcal{U}, \tilde{\boldsymbol{\sigma}} \in \Im, \boldsymbol{\theta} \in \mathcal{A}, \tilde{\boldsymbol{u}}=\tilde{\boldsymbol{u}}^{k}, k \in \wp,
\end{array}
$$

where $\tilde{\boldsymbol{u}}^{k}$ is the pseudo harmonic displacement response approximated by the measured displacement/acceleration auto-power spectrum density $S_{u u}^{k} / S_{a a}^{k}$ of the $k$ th structural response and $\{k \in \wp\}$ is the set of points measured by limited number of sensors. The approximated pseudo harmonic response $\tilde{\boldsymbol{u}}^{k}$ at position $k$ can be expressed as

$$
\begin{aligned}
\tilde{\boldsymbol{u}}^{k} & =(\boldsymbol{c}+\boldsymbol{d} i)^{k} \sqrt{S_{u u}^{k}(\omega)}=(\boldsymbol{c}+\boldsymbol{d} i)^{k} \sqrt{S_{a a}^{k}(\omega) / \omega^{4}} \\
& \approx\left(\mathbf{H}\left(\boldsymbol{\theta}_{(j)}\right) \boldsymbol{a}\right)^{k} \sqrt{S_{0}(\omega)}=\left(\left[-\omega^{2} \mathbf{M}+i \omega \mathbf{C}\left(\boldsymbol{\theta}_{(j)}\right)+\mathbf{K}\left(\boldsymbol{\theta}_{(j)}\right)\right]^{-1} \boldsymbol{a}\right)^{k} \sqrt{S_{0}(\omega)}
\end{aligned}
$$

where $\left(c^{k}\right)^{2}+\left(d^{k}\right)^{2}=1$ and the ratio $c^{k} / d^{k}$ is equal to the ratio of the real part to imaginary part of $\left(\mathbf{H}\left(\boldsymbol{\theta}_{(j)}\right) \boldsymbol{a}\right)^{k}$. $\boldsymbol{\theta}_{(j)}$ is the value of the damage parameter at $j$ th iteration step. This procedure is described in detail in Table 1.

\section{Basic concept of the identification strategy and main steps of the method}

\subsection{Stress field building strategy}

Consider an admissible stress field $\tilde{\boldsymbol{\sigma}}$ which satisfies the equilibrium equation as follows:

$$
\begin{aligned}
\mathbf{K} \tilde{\boldsymbol{u}} & =\omega^{2} \mathbf{M} \tilde{\boldsymbol{u}}-i \omega \mathbf{C} \tilde{\boldsymbol{u}}+\boldsymbol{a} \sqrt{S_{0}(\omega)}=\mathbf{L} \tilde{\boldsymbol{\sigma}} \\
\Rightarrow \mathbf{L} \tilde{\boldsymbol{\sigma}} & =\left(1+i \omega a_{2}\right)^{-1}\left[\left(\omega^{2}-i \omega a_{1}\right) \mathbf{M} \tilde{\boldsymbol{u}}+\boldsymbol{a} \sqrt{S_{0}(\omega)}\right] .
\end{aligned}
$$


Remark 1. For $\mathbf{A} \in \mathbb{R}^{n \times r}$ and $\operatorname{rank}(\mathbf{A})=n$ ( $\mathbf{A}$ is of full-row rank), consider a system of linear equations

$$
\mathbf{A} \boldsymbol{x}=\boldsymbol{b}
$$

If $n=r$, Eq. (13) admits a unique solution $\boldsymbol{x}=\mathbf{A}^{-1} \boldsymbol{b}$. In general, for $n<r$, a vector $\boldsymbol{x}$ that solves the system may not be unique. A computationally simple and accurate way to solve this equation is by using the SVD. That is, if $\mathbf{A}=\mathbf{U} \mathbf{\Sigma} \mathbf{V}^{*}$ is the $S V D$ of $\mathbf{A}$, the solutions of Eq. (13) are all given by

$$
\boldsymbol{x}=\mathbf{A}^{+} \boldsymbol{b}+\mathbf{Z} \boldsymbol{p}
$$

where $\mathbf{A}^{+}=\mathbf{V} \mathbf{\Sigma}^{+} \mathbf{U}^{*}$ is the pseudoninverse of matrix $\mathbf{A}, \boldsymbol{p}$ is an arbitrary column vector of length $r-n, \mathbf{Z}=\left\|\mathbf{A}^{+}\right\|_{\ell_{2}} \mathbf{V}_{2}$ and $\mathbf{V}=\left[\left(\mathbf{V}_{1}\right)_{r \times n},\left(\mathbf{V}_{2}\right)_{r \times(r-n)}\right] .\left\|\mathbf{A}^{+}\right\|_{\ell_{2}}$, denoting the $\ell_{2}$-norm of the matrix $\mathbf{A}^{+}$, is introduced to reduce the possible ill-conditioning.

Combining of Eq. (12) and Eq. (14) and setting $\mathbf{A}=\mathbf{L}, \boldsymbol{x}=\tilde{\boldsymbol{\sigma}}$ yields

$$
\tilde{\boldsymbol{\sigma}}=c_{2} c_{1} \mathbf{A}^{+} \mathbf{M} \tilde{\boldsymbol{u}}+c_{2} \mathbf{A}^{+} \boldsymbol{a} \sqrt{S_{0}(\omega)}+\mathbf{Z} \boldsymbol{p}
$$

where $c_{1}=\left(\omega^{2}-i \omega a_{1}\right)$ and $c_{2}=\left(1+i \omega a_{2}\right)^{-1}$. It should be pointed out that only the vector $\boldsymbol{p}$ is unknown in Eq. (15). As a result, to acquire the stress field $\tilde{\boldsymbol{\sigma}}$ is equal to acquire the vector $\boldsymbol{p}$. The objective function could be reformulated into

$$
e_{\mathrm{CRE}}(\tilde{\boldsymbol{u}}, \boldsymbol{p}, \boldsymbol{\theta})=\frac{1}{2} \int_{\omega_{\min }}^{\omega_{\max }}\left\|\mathbf{D}(\boldsymbol{\theta})^{-\frac{1}{2}}\left(\mathbf{D}(\boldsymbol{\theta}) \mathbf{B} \tilde{\boldsymbol{u}}-\left(c_{2} c_{1} \mathbf{A}^{+} \mathbf{M} \tilde{\boldsymbol{u}}+c_{2} \mathbf{A}^{+} \boldsymbol{a} \sqrt{S_{0}(\omega)}+\mathbf{Z} \boldsymbol{p}\right)\right)\right\|^{2} z(\omega) d \omega
$$

\subsection{Sparse regularization}

Usually, the amount of the measured data $S_{u u}^{k} / S_{a a}^{k}$ is insufficient due to the incomplete finite sensors and limited sample sources. This drives the inverse problem ill-posed and very sensitive to the measurement noise. To circumvent the ill-posedness and improve the robustness, the sparse regularization is used. The main prerequisite for the introduction of sparsity is that damages occur at a few locations and no other information regarding the damage is required. 
By incorporating the sparse regularization into Eq. $(10),\left(\mathrm{P}_{0}\right)$ can be replaced by $\left(\mathrm{P}_{s}\right)$

$$
\begin{array}{cl}
\left(\mathrm{P}_{s}\right) & (\tilde{\boldsymbol{u}}, \boldsymbol{p}, \boldsymbol{\theta})=\arg \min e_{\mathrm{CRE}}(\tilde{\boldsymbol{u}}, \boldsymbol{p}, \boldsymbol{\theta})+\lambda\left\|\boldsymbol{\theta}-\boldsymbol{\theta}_{0}\right\|_{\ell_{1}}^{1} \\
& \text { subject to } \tilde{\boldsymbol{u}} \in \mathcal{U}, \tilde{\boldsymbol{\sigma}}(\boldsymbol{p}) \in \Im, \boldsymbol{\theta} \in \mathcal{A}, \tilde{\boldsymbol{u}}=\tilde{\boldsymbol{u}}^{k}, k \in \wp,
\end{array}
$$

where $\lambda$ is the non-negative sparse regularization parameter controlling the trade-off between the sparsity and CRE, $\left\|\boldsymbol{\theta}-\boldsymbol{\theta}_{0}\right\|_{\ell_{1}}^{1}=\sum_{e=1}^{m}\left|\theta_{e}-\theta_{0 e}\right|$ and $\theta_{0 e}:=1$ for the eth element of the initial intact structure. Large value of $\lambda$ makes the number of the nonzeros in $\boldsymbol{\theta}-\boldsymbol{\theta}_{0}$ as scarce as possible. According to previous research, the 'threshold setting method' (TSM) is used to estimate the optimal regularization parameter where $\lambda_{\text {est }}=\operatorname{TSM}\left(\Lambda, l_{\max }, \alpha\right)$. For more details, refer to the work [13].

\subsection{Alternating minimization (AM) method}

The alternating minimization $(\mathrm{AM})$ method is proposed for the program $\left(\mathrm{P}_{s}\right)$ due to the separate convexity property of the objective function of this problem. To this end, no sensitivity analysis is required herein. The AM method is an iterative approach and try to find the solution in each iteration by alternating minimization of the objective function over separated variables. With an initial guess of the damage parameters $\boldsymbol{\theta}_{0}$, the solving process is described below.

- step 1 (UMF step): Update the mechanical field $(\tilde{\boldsymbol{u}}, \boldsymbol{p})$ with the given damage parameters $\boldsymbol{\theta}_{(j-1)}$

$$
\left(\tilde{\boldsymbol{u}}_{(j)}, \boldsymbol{p}_{(j)}\right)=\arg \min _{u \in \mathcal{U}, \boldsymbol{\sigma}(\boldsymbol{p}) \in \Im, u=\hat{u}^{k}, k \in \wp} e_{\mathrm{CRE}}\left(\tilde{\boldsymbol{u}}, \boldsymbol{p}, \boldsymbol{\theta}_{(j-1)}\right)
$$

- step 2 (UDP step): Update the damage parameters $\boldsymbol{\theta}$ by the mechanical fields $\left(\tilde{\boldsymbol{u}}_{(j)}, \boldsymbol{p}_{(j)}\right)$ obtained in step 1.

$$
\boldsymbol{\theta}_{(j)}=\arg \min _{\boldsymbol{\theta} \in \mathcal{A}} e_{\mathrm{CRE}}\left(\tilde{\boldsymbol{u}}_{(j)}, \boldsymbol{p}_{(j)}, \boldsymbol{\theta}\right)+\lambda\left\|\boldsymbol{\theta}-\boldsymbol{\theta}_{0}\right\|_{\ell_{1}}^{1}
$$

For mathematical proof on the convergence of the AM method, the reader is referred to the reference [27]. The detailed expressions for each step are shown in Appendix.

\subsection{Summary of the proposed damage identification approach}

By combining the UMF step and the UDP step in Section 3.3 and using the threshold setting method so as to estimate the optimal regularization parameter $\lambda$, the damage identification algorithm by min-CRE and sparse regularization based on power spectrum density data can be established, as shown in Table 1. 
Table 1 Summery of the proposed AM method

Initial algorithm setting

-set initial damage parameters $\boldsymbol{\theta}_{(0)}=\boldsymbol{\theta}_{0}$ as those of the intact structure

-set values of the maximum threshold setting rank $l_{\max }$ and the discriminating ratio

$\alpha$ for the threshold setting method [13]

-define the convergence tolerance TOL and the maximum number of iterations $Z_{\max }$ -load the measured response power spectrum density data $S_{u u}^{k} / S_{a a}^{k}$ as well as the excitation power spectrum density data $S_{0}(\omega)$

-construct the pseudo harmonic excitation $\boldsymbol{a} \sqrt{S_{0}(\omega)}$ (section 2.1)

-assemble the matrix $\mathbf{L}$ and $\mathbf{R}$ and refer to Remark 1 to get the matrices $\mathbf{A}$ and $\mathbf{Z}$

Alternating minimization approach (for $j=1: Z_{\max }$ )

-assemble the mass, stiffness and frequency response matrices $\mathbf{M}, \mathbf{K}$ and $\mathbf{H}$ using current value of $\boldsymbol{\theta}_{(j-1)}$

-obtain the approximated pseudo harmonic response $\boldsymbol{u}_{(j)}^{k}=(\boldsymbol{c}+\boldsymbol{d} i)^{k} \sqrt{S_{u u}^{k}(\omega)}(\mathrm{sec}-$ tion 2.2)

-refer to the UMF step to obtain the updating mechanical field (section 3.3, Appendix A.2)

$$
\left(\begin{array}{c}
\tilde{\boldsymbol{u}}_{(j)} \\
\boldsymbol{p}_{(j)}
\end{array}\right)=\left(\begin{array}{c}
\tilde{\boldsymbol{u}}_{(j)}^{k} \\
\left(\mathbf{R}^{U U}\right)^{-1}\left(\boldsymbol{S}^{U}-\mathbf{R}^{U M} \tilde{\boldsymbol{u}}_{(j)}^{k}\right)
\end{array}\right)
$$

-choose the optimal regularization parameter $\lambda$ according to the threshold setting method [13]

-refer to the UDP step to obtain the updating damage parameter $\theta_{e(j)}, e=1, \cdots, m$ (section 3.3, Appendix A.3):

- if $\lambda<\frac{1}{2}\left(\frac{b_{e}}{\theta_{0 e}^{2}}-a_{e}\right)$, then $\theta_{e(j)}=\sqrt{\frac{b_{e}}{a_{e}+2 \lambda}}$,

- else if $\lambda<\frac{1}{2}\left(a_{e}-\frac{b_{e}}{\theta_{0 e}^{2}}\right)$, then $\theta_{e(j)}=\sqrt{\frac{b_{e}}{a_{e}-2 \lambda}}$,

- else $\theta_{e(j)}=\theta_{e 0}=1$

-Convergence criterion

- if $\left\|\tilde{\boldsymbol{\theta}}_{(j)}-\tilde{\boldsymbol{\theta}}_{(j-1)}\right\| /\left\|\tilde{\boldsymbol{\theta}}_{(0)}\right\| \leq$ TOL break 
(a)

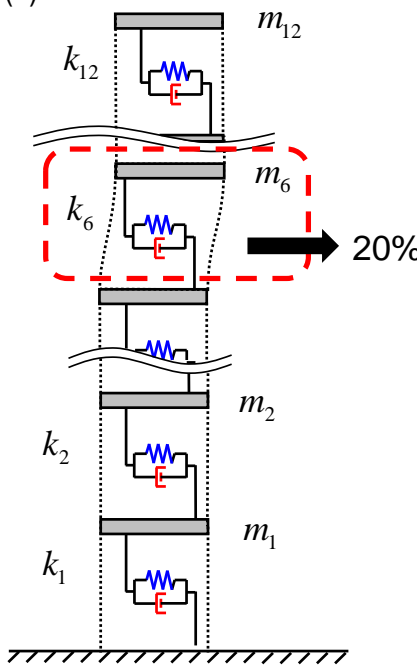

D1: single damage

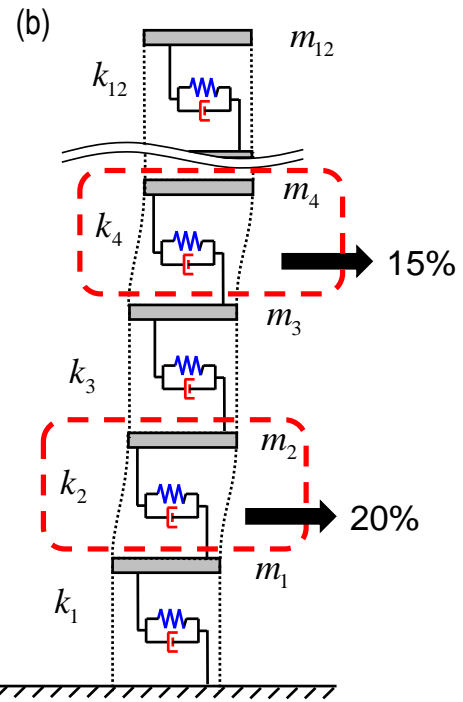

D2: multiple damages

Figure 1 Overview of the numerical model

\section{Numerical verification}

A 12-story shear building structure as shown in Figure $\mathbf{1}$ is used to test the proposed damage identification approach first (see reference [28] for details of the structure). The mass and stiffness parameters of the intact system are $m_{1}=m_{2}=\cdots=m_{12}=1.0 \times 10^{5} \mathrm{~kg}, k_{1}=k_{2}=$ $\cdots=k_{12}=2.0 \times 10^{8} \mathrm{~N} / \mathrm{m}$. The Rayleigh damping model is adopted with the damping ratios taken equal to $5 \%$ and the Rayleigh coefficients $\alpha$ and $\beta$ are 0.506 and $1.073 \times 10^{-4}$, respectively. Two damage cases are considered: Single damage (D1), the stiffness of the 6th story is reduced by $20 \%\left(\theta_{6}=0.8 /\right.$ stiffness reduction $\left.=0.2\right)$; Multiple damages (D2), the stiffness of 2 nd story and 4 th story is reduced by $5 \%$ and $15 \%\left(\theta_{2}=0.95, \theta_{4}=0.85 /\right.$ stiffness reduction $\left.=0.05,0.15\right)$, respectively.

Assume that the structure is subjected to a stationary acceleration excitation of white noise from the ground. The measured accelerations of the 1st and 2nd stories ( $a_{1}$ and $\left.a_{2}\right)$ are taken from the results of numerical simulation during the time period $[0,60 s]$ with a sampling rate of $500 \mathrm{~Hz}$ and polluted by a standard normally distributed random noise of $10 \%$ level (Figure 2). Figure 3 shows the comparison of the power spectrum density between the initial (undamaged) model and the damaged model at the 2nd story for D1.

Identification results for respective damage scenarios of the proposed approach are summarized in Figure 4 (a) and (b). As can be seen, damage is identified successfully for the single damage case D1 with an optimal value of regularization parameter $\lambda=1.211 \times 10^{-11}$. Regarding 


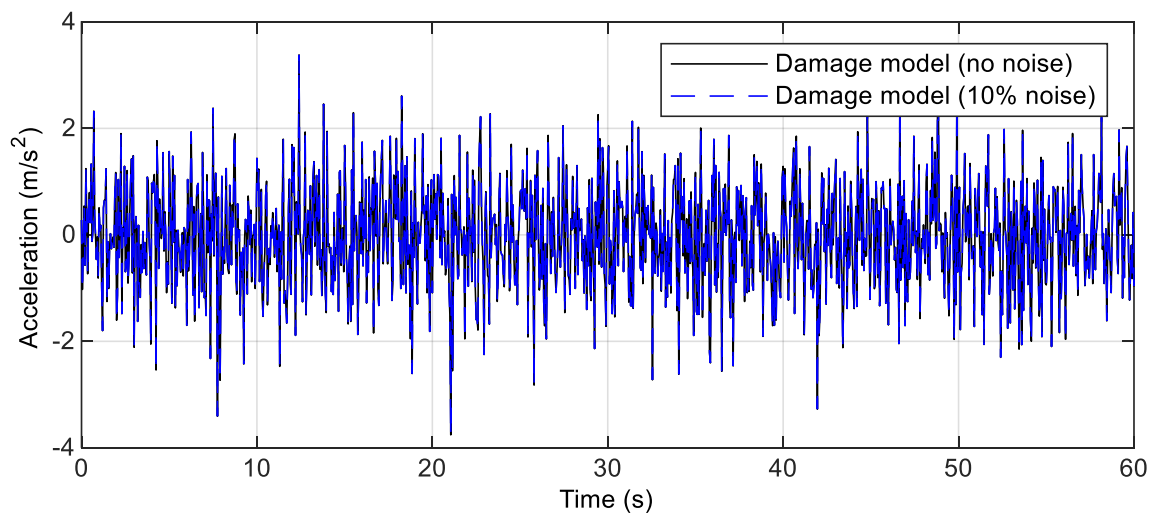

Figure 2 Response at 2nd floor for D1

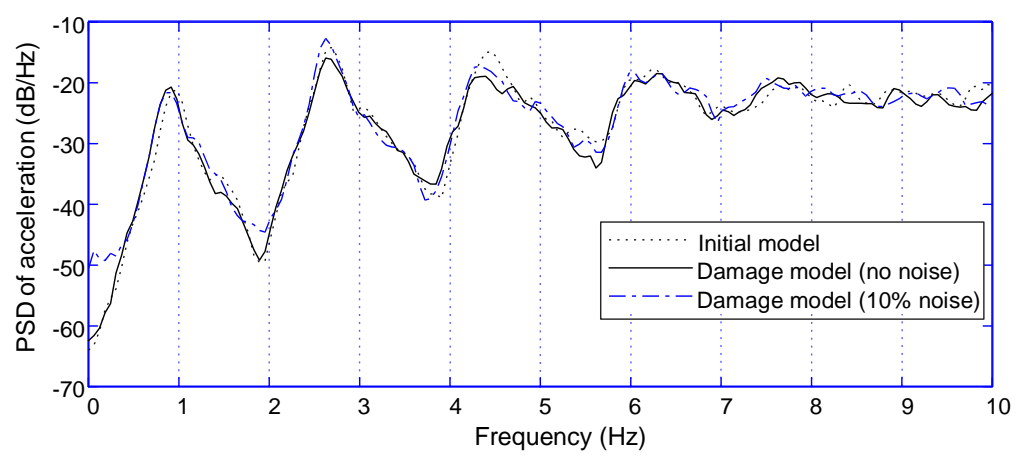

Figure 3 Power spectrum density of acceleration at 2nd floor for D1 


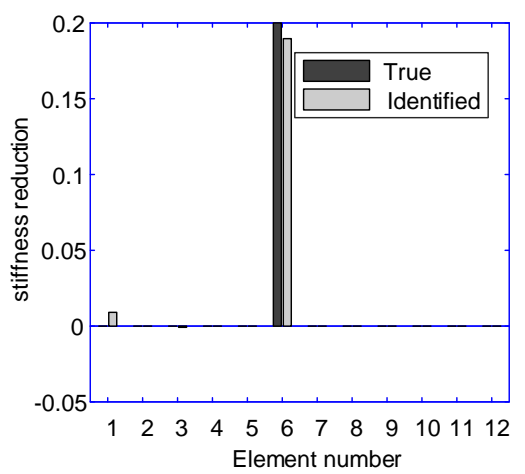

(a)

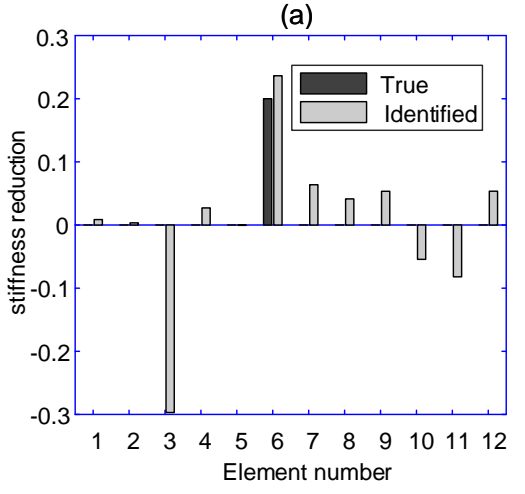

(c)

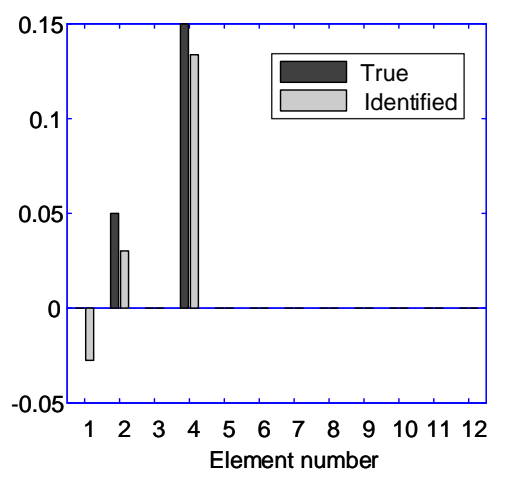

(b)

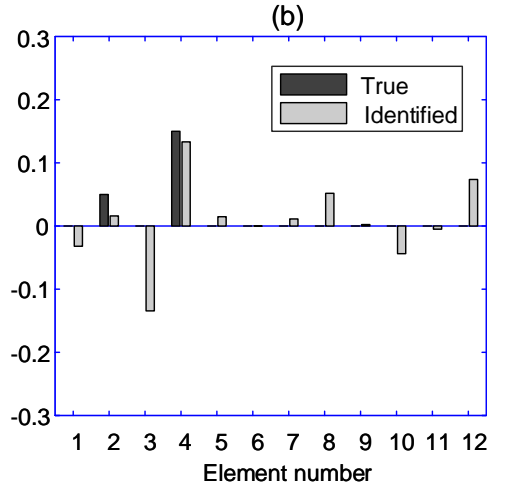

(d)

Figure 4 Identification results with 10\% noise: (a) D1 with sparsity, (b) D2 with sparsity, (c) D1 without sparsity and (d) D2 without sparsity

the case D2, the multiple damages case seems to be more sensitive to the noise: some unwanted yet acceptable stiffness change at the 1st story was also observed. This phenomenon is due to the different effectiveness of the sparse regularization [13]. Sparsity obviously has a pronounced effect on less number of damages while multiple damages reduce sparsity in intensity. The optimal sparse regularization parameter is found to be $1.018 \times 10^{-11}$ for D2. Figure 4 (c) and (d) demonstrate the identification results for D1 and D2 without the enforcement of sparsity. One can find that it is almost impossible to get to the reasonable identification results when measurement data are limited and noise level is relatively high. To this end, the effectiveness of the sparse regularization has been proved.

To further validate the proposed approach, two generally used identification techniques: the sensitivity-based algorithm with Tikhonov regularization $\left(\ell_{2}\right.$ norm) and the Unscented Kalman Filter (UKF) are additionally conducted for the damage case D1 as comparisons in the following. Acceleration/Displacement measurements in time domain are directly used for identification in both of these methods. Detailed discussion of the theoretical background of these two methods is beyond the scope of this paper. Table 2 briefly outlines the basic information of these methods. 
Table 2 Basic information of the sensitivity-based algorithm with Tikhonov regularization and the UKF

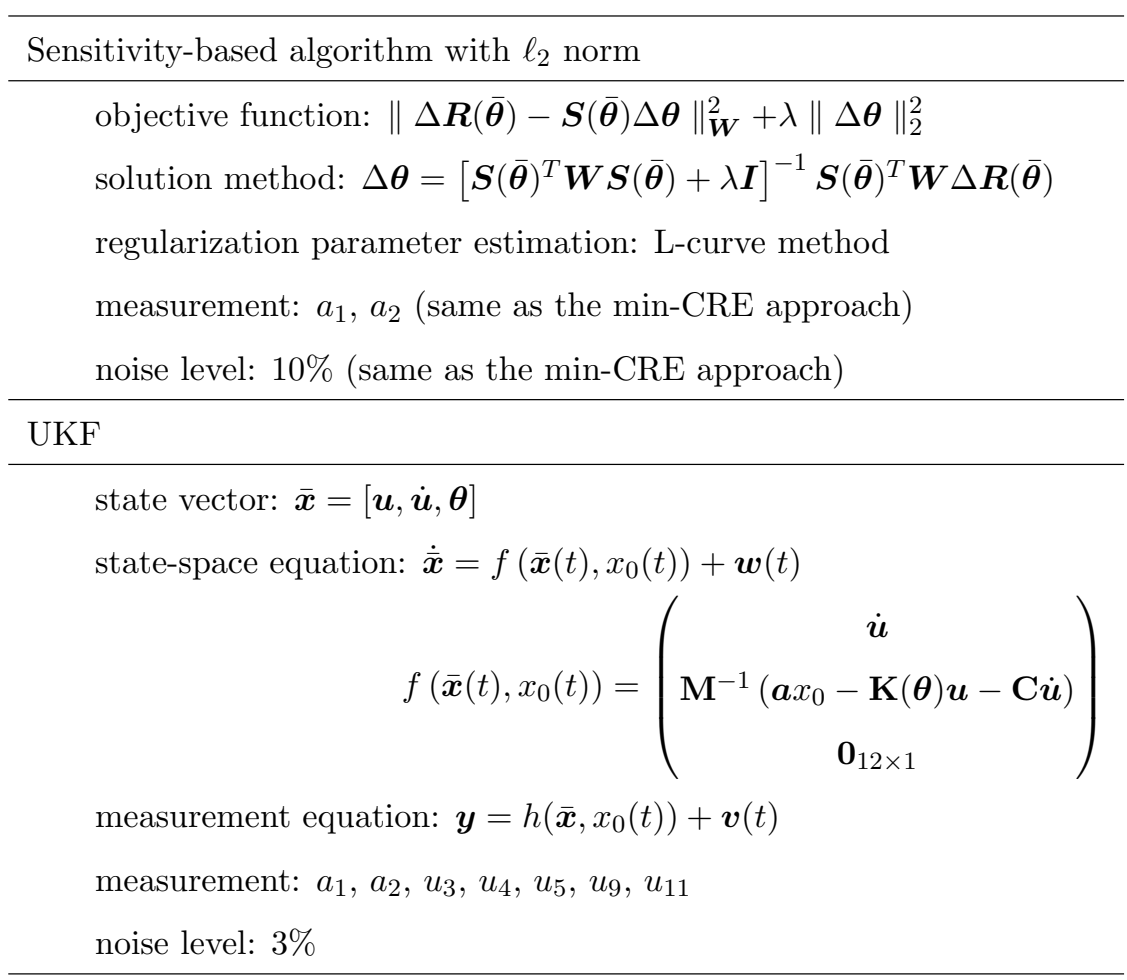

More technical details can be found in $[2,29]$ and [30, 31] respectively.

The results presented in Figure $\mathbf{5}$ show the successful performance of both of the two methods. On one hand, the sensitivity-based algorithm could well identify the damage location and extent using the same limited measurements at the same noise level, as the proposed min-CRE approach. The difference lies in the effects of different regularization enforcements. Attention should be paid on that damages can be observed at some false positions with minor degree (Figure $5(\mathrm{a})$ : element $7,9,10,12)$ for the use of Tikhonov regularization. This is due to the fact that the sparse regularization finds the correct sparse solution while the Tikhonov regularization tends to produce a smoother but not accurate enough solution. For comprehensive study about this difference, the reader is referred to [32].

One the other hand, measurement positions have to be increased while noise level goes down so that the UKF could give satisfied identification results, as shown in Table 2. The fact that newly added measurements are chosen to be displacements $\left(u_{3}, u_{4}, u_{5}, u_{9}, u_{11}\right)$ instead of accelerations is due to the reason that identification results from the UKF could be much improved when fusing displacement measurements are included [30]. Performance of the parameter estimation changed with time is plotted in Figure 6. Despite of the aforementioned limitations, the 


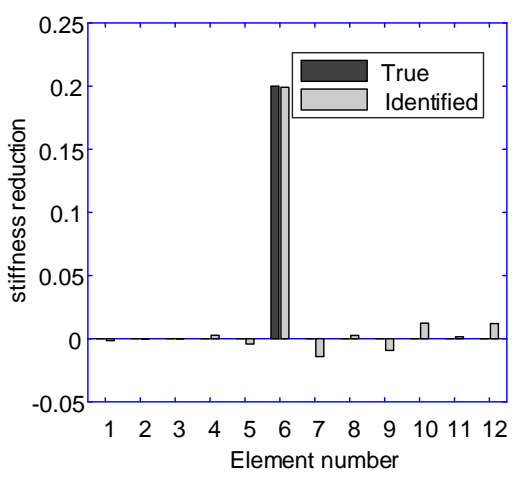

(a)

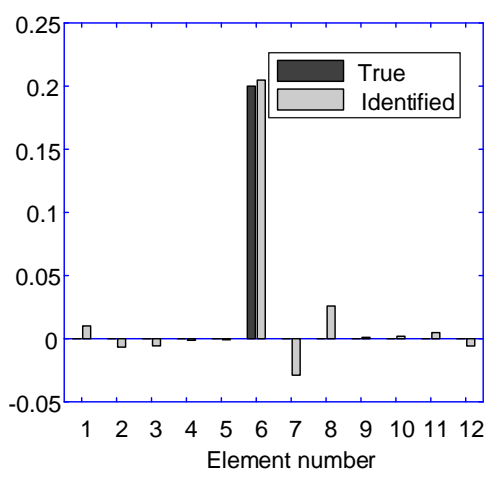

(b)

Figure 5 Identification results using different methods: (a) sensitivity-based algorithm, (b) $\mathrm{UKF}$

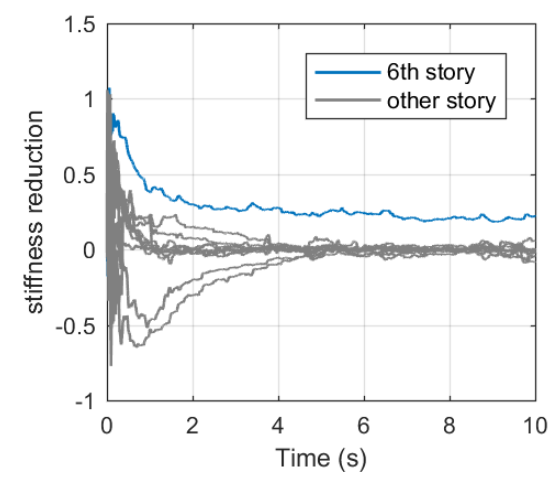

Figure 6 Parameter estimation performance using UKF

UKF is still proven to be an attracting method because of its ability to quantify the uncertainty in the parameters, convenience in the process of equating as well as the on-line tracking nature.

\section{Experimental verification}

In this section, the experimental verification of using the proposed min-CRE approach for damage identification will be further presented for a laboratory plane frame structure. The experimental set up, data generation, numerical models and damage identification results will be described in details in the following.

\subsection{Experimental setup and data generation}

Two two-story testing plane frames, undamaged frame (UF) and damaged frame (DF), are fabricated in the structure laboratory in Kyoto University, as shown in Figure 7(a). Parameters of these frames are shown in Table 3 . 
Table 3 Characteristics of the two-story testing plane frames

In $\mathrm{DF}$, damages are introduced by reducing the rectangular cross section in the area of left $1 / 2$ length of the beam in the first story, from $b_{1} \times h_{1}=40 \mathrm{~mm} \times 1 \mathrm{~mm}$ to $b_{d} \times h_{1}=20 \mathrm{~mm} \times 1 \mathrm{~mm}$, as illustrated in Figure 7 (b). As a result, the approximated stiffness reduction $\left(k^{\text {damage }} / k_{0}\right)$ for this area is estimated as 0.5. For the sake of clarity, it should be noted that in this example, since the mass matrix of the structure is mainly determined by the lumped mass instead of the beam, only the change of stiffness is identified while the change of mass is ignored.

The frame is bolted on the shaking table and tested using ground excitation (Figure $7(d)$ ). The sampling frequency is set to $200 \mathrm{~Hz}$ and nine accelerometers(A0-A8) are installed for this test (Figure 7(a), Figure 8). Each accelerometer is weighted about $0.02 \mathrm{~kg} \sim 0.03 \mathrm{~kg}$. The horizontal acceleration responses are measured during the time period $[0,30] \mathrm{s}$.

\subsection{Numerical baseline model}

An initial finite element (FE) model is established according to the geometric and physical properties of the experimental UF, with 42 planar elements and 42 nodes as shown in Figure 8. Each node has three DOFs (two transnational displacements and one rotational displacement) and the system has 126 DOFs in total. The weights of lumped masses are added to the corresponding nodes and adjacent beam elements are set as rigid link in the FE model.

The UF was tested first using white noise ground excitation. Natural frequencies and mode shapes of the frame are then determined through the subspace identification algorithm (n4sid). The subspace approach to state-space modeling offers numerically reliable algorithms for com- 


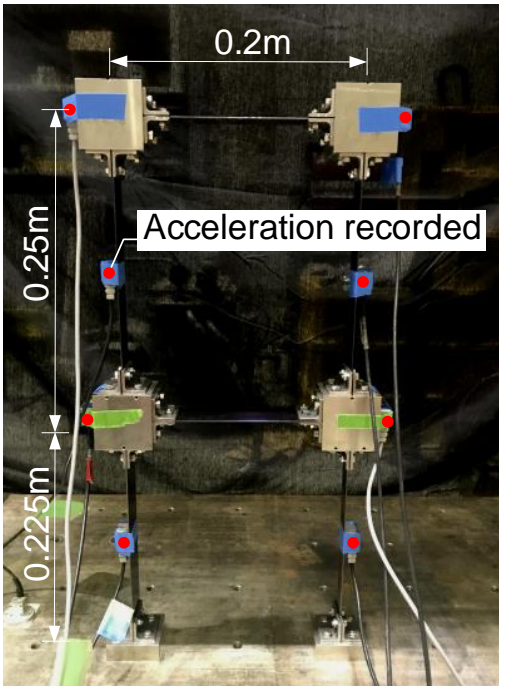

(a)

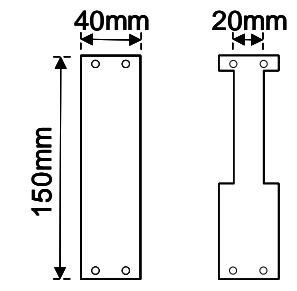

undamaged damaged

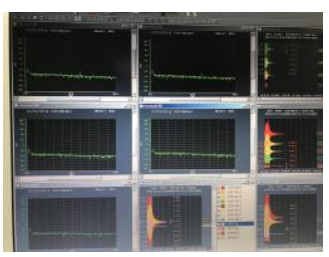

(c)

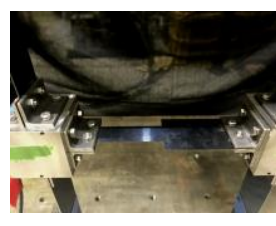

(b)

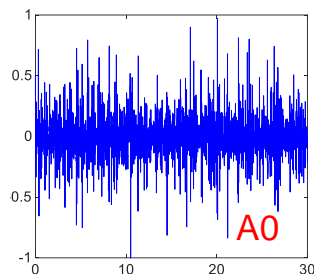

(d)

Figure 7 Overview of the test model: (a) test frame (UF/DF), (b) undamaged and damaged beam, (c) data acquisition, (d) acceleration of the ground excitation: white noise
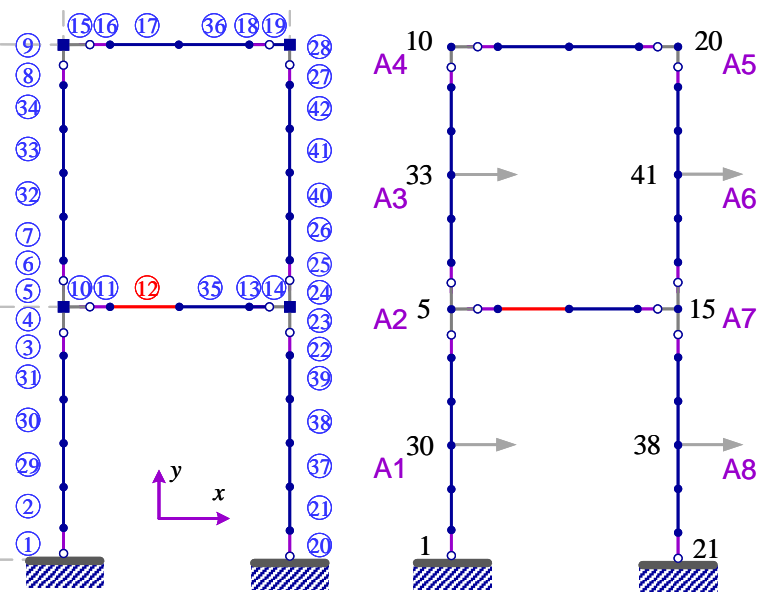

Node numbers:

$1,4, \cdots, 42$

Element numbers:

(1), 2), $\cdots, 42$

Damaged elements: (12)

Measured direction:

Rigid link: -

L-angle:

Lumped mass: -

Accelerometers:

A1-A8

Figure $8 \mathrm{FE}$ Model of plane frame structure 
Table 4 Natural frequencies of the baseline model, experimental UF and Experiment DF

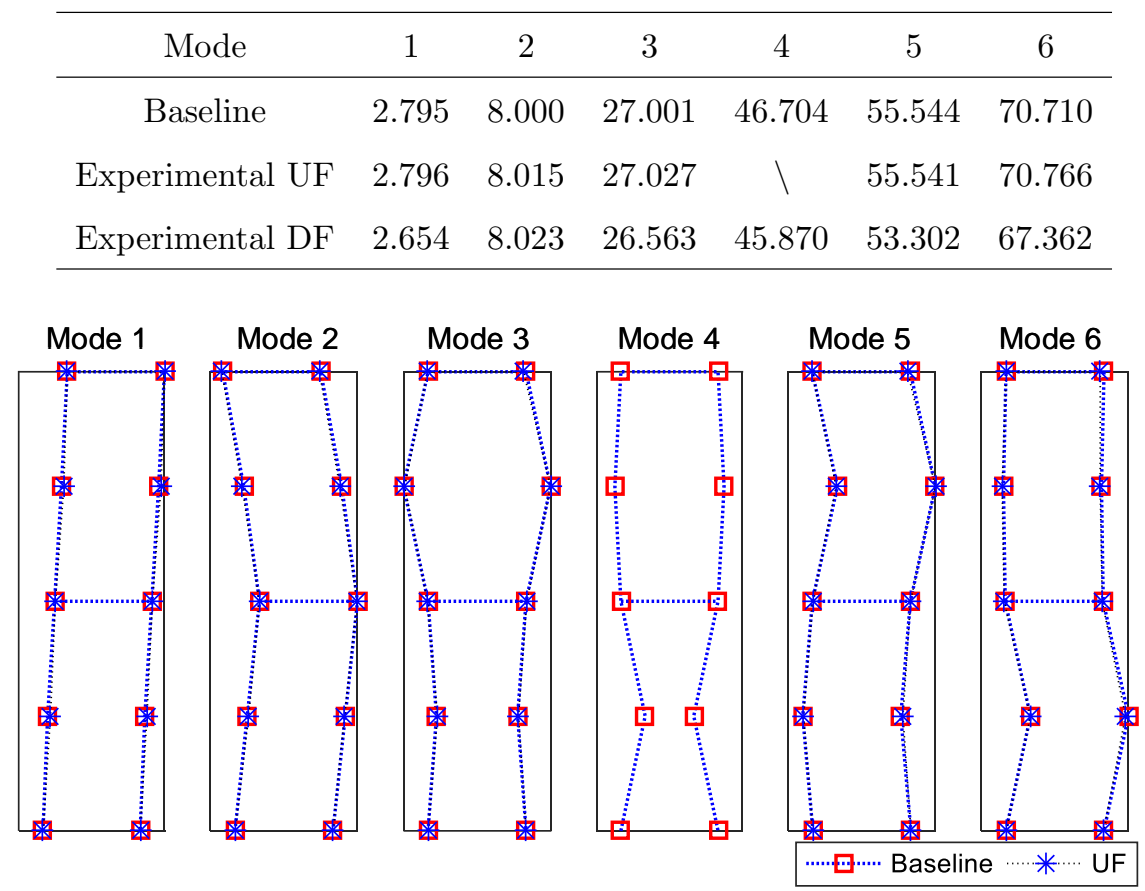

Figure 9 Mode shapes of the experimental UF and the baseline model

puting state-space descriptions directly using measured input-output data. For technical details on this method, the reader is referred to [33, 34]. The obtained modal data are used to perform the initial model updating to minimize the difference between the experimental UF and the FE model by the sensitivity-based method. This procedure was described in detail in reference [35]. Table 4 and Figure 9 show that the natural frequencies and mode shapes predicted by the updated FE model (the baseline model) match the experimental test results fairly well. It should be pointed out that the 4th mode was not identified through the experiment data and comparisons of this mode could not be conducted consequently.

A further validation of the updated model was performed by comparing the experimental accelerations and those obtained by the updated FE model (Figure 10). As the FE model is only identified based on the experimental modal data, the identified damping parameters using the Rayleigh damping assumption are used in this comparison. The Rayleigh coefficients $\alpha$ and $\beta$ are 0.7102 and $6.9325 \times 10^{-5}$, respectively. So far, the accuracy of the FE modal was strongly verified. This updated FE model is then used as the baseline model in the following damage identification process. 


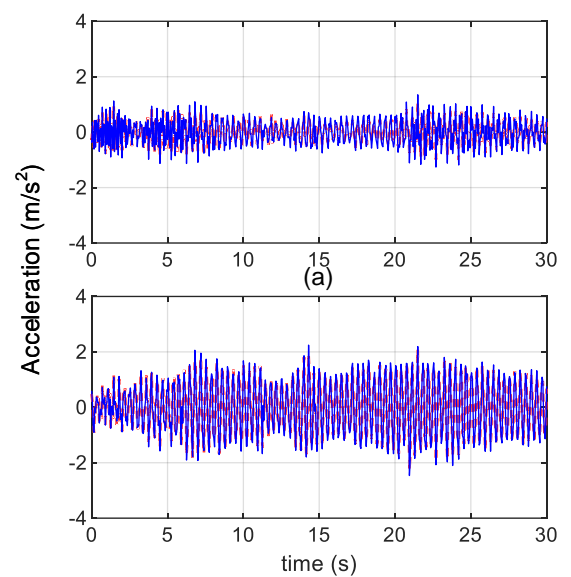

(c)

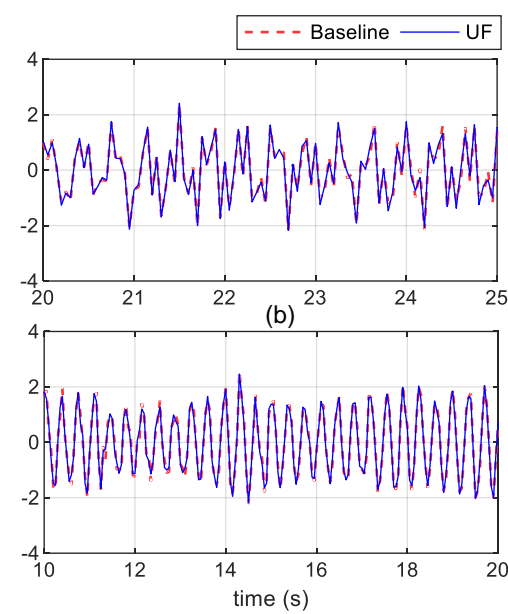

(d)

Figure 10 Accelerations of the experimental UF model and the baseline model at: (a) A1, (b) $\mathrm{A} 2$, (c) A3 and (d) A4

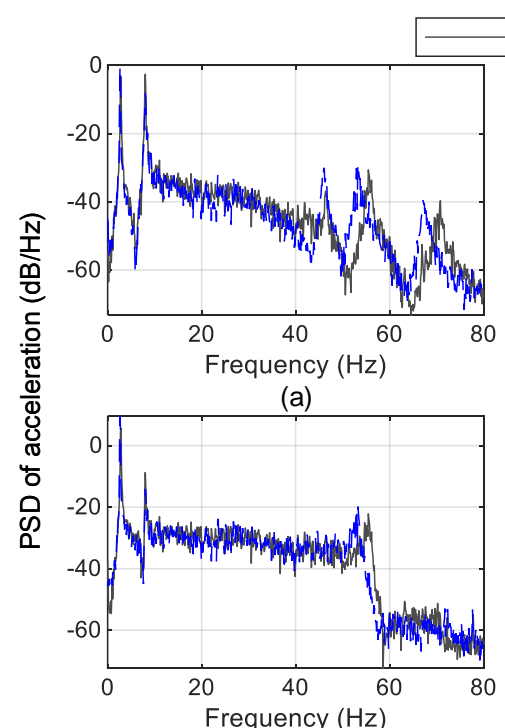

(c)
Experimental UF - - Experimental DF

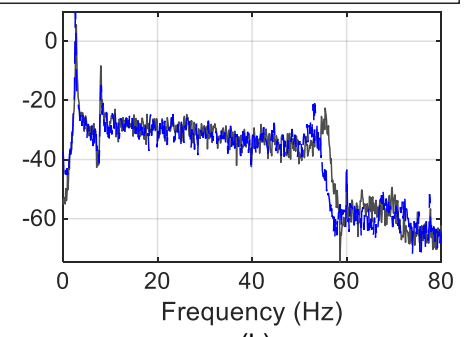

(b)

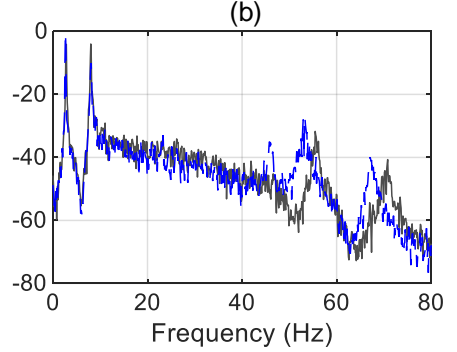

(d)

Figure 11 Measured PSD comparison between the experimental UF and the experimental DF:

(a) A1, (b) A3, (c) A6 and (d) A8 

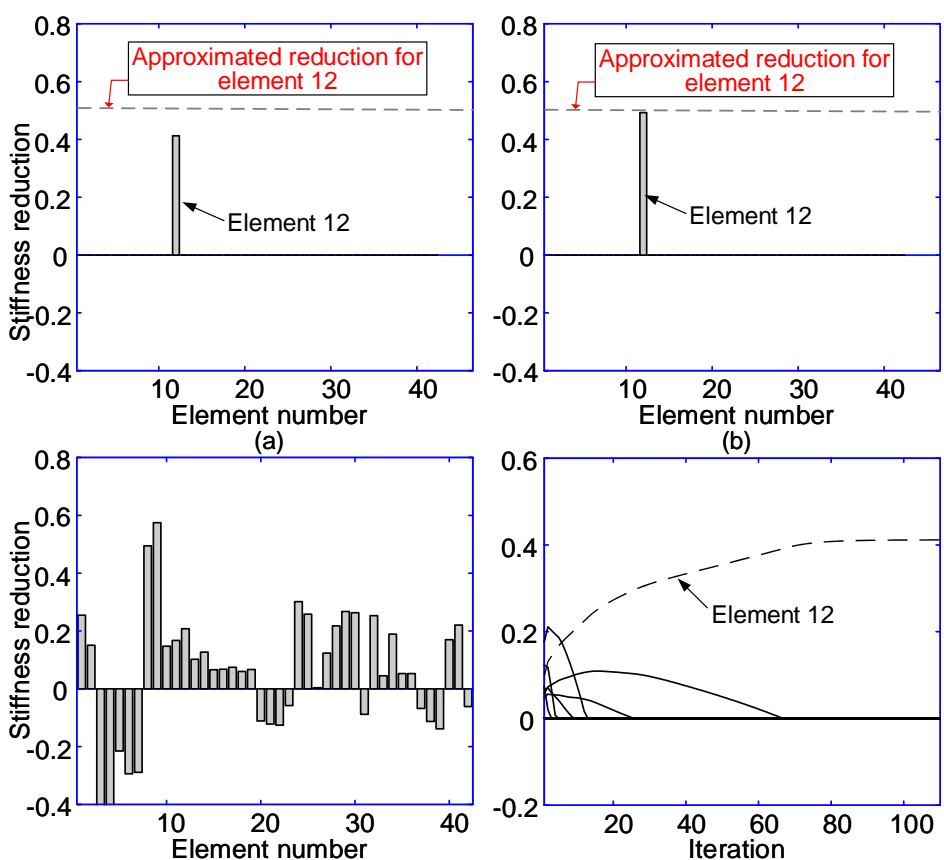

(c)

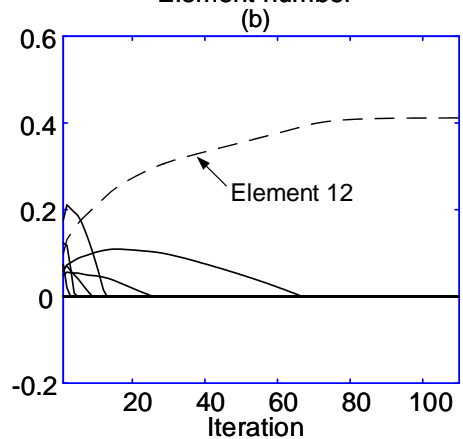

(d)

Figure 12 Identified results: (a) G1 (b) G2 (c) G1 without sparsity (d) stiffness reduction vs iteration in case G1

\subsection{Damage identification}

In the next identification step, the experimental DF is further subjected to the white noise excitation from the ground. Accelerations are obtained and then transformed into power spectrum densities using the Welch's method. To improve the robustness and efficiency of the identification approach, data pre-selection is conducted. A specific frequency range of $[45,72] \mathrm{Hz}$ is selected for analysis due to the fact that obvious differences in the power spectrum density data in A1, A3, A6 and A8 within this frequency range can be observed before and after damage, as shown in Figure 11.

Using the measured data from the accelerometers installed at all positions is not necessary for the proposed identification approach. As a result, only accelerometers A1, A3, A6 and A8 are used for damage identification. To investigate the data from which accelerometers are more efficient for identification, the accelerometers are divided into two groups: (1) G1: A1\&A8 and (2) G2: A3\&A6.

Identification results are shown in Figure 12. As could be seen, the proposed approach with sparse regularization could identify the damage location (Element 12) clearly. Compared to G1, G2 results in more satisfactory damage extent assessment. The optimal regularization parameters are $\lambda=3.074 \times 10^{-12}$ for G1 and $\lambda=1.980 \times 10^{-12}$ for G2. Figure $12(\mathrm{c})$ is 


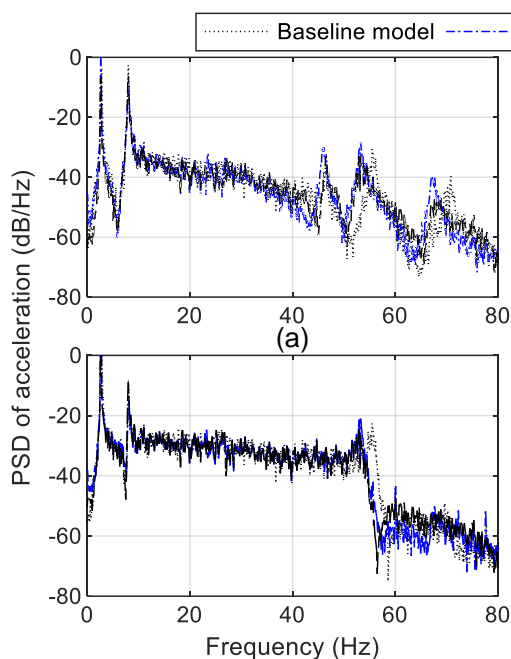

(c)
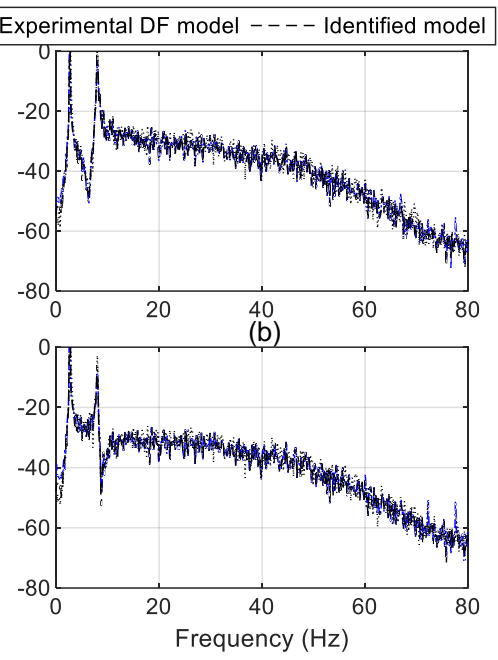

(d)

Figure 13 Power spectrum density of acceleration at: (a)A1, (b)A2, (c)A3 and (d)A4

concerned with the identified results without any sparse regularization for G1. As is observed, neither damage location nor damage extent could be appropriately identified in such situation. Sparse regularization has demonstrated a pronounced effect on the accuracy and robustness for the identification process. To testify the convergence of the proposed approach, the number of iterations is illustrated in Figure 12(d). One can find that damage is clearly identified after 100 steps. This result verifies the convergence of the proposed method.

Although the proposed identification approach is a procedure of updating damage parameter of the identified model to best fit the constitutive relation, rather than the dynamic response of the experimental structure, the power spectrum densities of the baseline beam model, identified model and experimental DF at A1-A4 are depicted in Figure 13. One can find that the power spectrum density of the identified model corresponds with the experimental DF well. This case shows that the proposed approach is as effective to identify the damage parameters as those methods which measure the response discrepancies between the numerical model and the dynamic test results.

\section{Conclusions}

Usage of the error in constitutive relation for damage identification has gained increasing attention from many engineers recently. An effective and simple solution procedure so as to use the $\mathrm{CRE}$ as the objective function is crucial to the development of this technique. A spectrum-driven damage identification strategy based on the min-CRE approach was proposed in this paper. The 
sparse regularization has been enforced to enhance the robustness of this approach with introducing little additionally computational effort due to the favorable mathematical features of the CRE objective function. The AM method was used to get the solution to this nonlinear optimization problem. The proposed method has the advantage that only measurement power spectrum density data from few limited sensors are needed for damage identification. Numerical and experimental verification examples show that the proposed method is insensitive to measurement noise and effective for both small and large damage identifications. Structures with damages occurring at less locations are recommended for the proposed approach. Extending this method to the large scale practical strictures will be discussed in the further research.

\section{Acknowledgement}

The present research was performed under the support of the MEXT scholarship of Japan.

\section{APPENDIX}

\section{A.1 Decomposition of the stiffness matrix}

Consider a structure occupying the domain $\Omega$ which is divided into $m$ elements, for each element $e$ in the field of $\Omega_{e}$, the element stiffness matrix can be written as

$$
\mathbf{K}^{e}=\int_{\Omega^{e}} \mathbf{B}_{e} \mathbf{D}_{e}^{\prime} \mathbf{B}_{e} d \Omega=\mathbf{L}_{e} \mathbf{D}_{e} \mathbf{R}_{e}
$$

The specific decomposition of the element stiffness matrix for 1D spring element and 2D EulerBernoulli beam element are given below.

- 1D spring element where $k_{i}$ denotes the stiffness of the $i$ th spring

$$
\mathbf{D}_{e}=k_{e}, \mathbf{R}_{e}=\left(\begin{array}{ll}
1 & -1
\end{array}\right)=\mathbf{L}_{e}^{\mathrm{T}} .
$$

- 2D Euler-Bernoulli beam element with length $h$, Young's modulus $E$, cross-sectional area $A$ and inertial moment $I$

$$
\mathbf{L}_{e}=\left(\begin{array}{ccc}
1 & 0 & 0 \\
0 & 0 & \frac{2 \sqrt{3}}{h} \\
0 & 1 & \sqrt{3} \\
-1 & 0 & 0 \\
0 & 0 & -\frac{2 \sqrt{3}}{h} \\
0 & -1 & \sqrt{3}
\end{array}\right), \quad \mathbf{D}_{e}=\left(\begin{array}{ccc}
E A & 0 & 0 \\
0 & E I & 0 \\
0 & 0 & E I
\end{array}\right), \quad \mathbf{R}_{e}=\left(\begin{array}{cccccc}
\frac{1}{h} & 0 & 0 & -\frac{1}{h} & 0 & 0 \\
0 & 0 & \frac{1}{h} & 0 & 0 & -\frac{1}{h} \\
0 & \frac{2 \sqrt{3}}{h^{2}} & \frac{\sqrt{3}}{h} & 0 & -\frac{2 \sqrt{3}}{h^{2}} & \frac{\sqrt{3}}{h}
\end{array}\right) .
$$


Define a generalized discrete stress $\boldsymbol{\sigma}^{e}=\mathbf{D}_{e} \mathbf{R}_{e} \boldsymbol{u}^{e}$, where $\boldsymbol{\sigma}^{e}$ is in the form of column matrix, and let the global stiffness matrix obtained by $\mathbf{K}=\sum_{e} \mathbf{T}^{e T} \mathbf{K}^{e} \mathbf{T}^{e}$, where $\mathbf{T}^{e}$ is the local assembly matrix mapping the global DOFs into the elemental DOFs. We can then derive the equation

$$
\begin{aligned}
\mathbf{K} \boldsymbol{u} & =\sum_{e} \mathbf{T}^{e \mathrm{~T}} \mathbf{K}^{e} \mathbf{T}^{e} \boldsymbol{u} \\
& =\sum_{e} \mathbf{T}^{e \mathrm{~T}} \mathbf{K}^{e} \boldsymbol{u}^{e} \\
& =\sum_{e} \mathbf{T}^{e \mathrm{~T}} \mathbf{L}_{e} \mathbf{D}_{e} \mathbf{R}_{e} \boldsymbol{u}^{e} \\
& =\sum_{e} \mathbf{T}^{e \mathrm{~T}} \mathbf{L}_{e} \boldsymbol{\sigma}^{e}=\left(\begin{array}{llll}
\mathbf{T}^{1 \mathrm{~T}} \mathbf{L}_{1} & \mathbf{T}^{2 \mathrm{~T}} \mathbf{L}_{2} & \cdots, & \mathbf{T}^{m \mathrm{~T}} \mathbf{L}_{m}
\end{array}\right)\left(\begin{array}{c}
\boldsymbol{\sigma}^{1} \\
\boldsymbol{\sigma}^{2} \\
\vdots \\
\boldsymbol{\sigma}^{m}
\end{array}\right)
\end{aligned}
$$

Let $\mathbf{L}$ denote $\left(\begin{array}{lllll}\mathbf{T}^{1 \mathrm{~T}} \mathbf{L}_{1} & \mathbf{T}^{2^{\mathrm{T}}} \mathbf{L}_{2} & \cdots, & \mathbf{T}^{m \mathrm{~T}} \mathbf{L}_{m}\end{array}\right)$ and $\boldsymbol{\sigma}$ denote $\left(\begin{array}{llll}\boldsymbol{\sigma}^{1} & \boldsymbol{\sigma}^{2} & \ldots, & \boldsymbol{\sigma}^{m}\end{array}\right)^{\mathrm{T}}$. The equation as described in Eq. (6) can be finally obtained.

\section{A.2 UMF step}

To see more into the UMF step Eq. (18), the objective function Eq. (16) is regrouped into

$$
\begin{gathered}
e_{\mathrm{CRE}}\left(\boldsymbol{x}, \boldsymbol{\theta}_{(j-1)}\right)=\frac{1}{2} \int_{\omega_{\min }}^{\omega_{\max }} \| \mathbf{D}\left(\boldsymbol{\theta}_{(j-1)}\right)^{-\frac{1}{2}}\left(\left(\mathbf{D}\left(\boldsymbol{\theta}_{(j-1)}\right) \mathbf{R}-c_{2} c_{1} \mathbf{A}^{+} \mathbf{M},-\mathbf{Z}\right) \boldsymbol{x}\right. \\
\left.-c_{2} \mathbf{A}^{+} \boldsymbol{a} \sqrt{S_{0}(\omega)}\right) \|^{2} z(\omega) d \omega
\end{gathered}
$$

where $\boldsymbol{x}=(\tilde{\boldsymbol{u}} ; \boldsymbol{p})$. For the sake of convenience, superscript $U$ and $M$ correspond to the unmeasured and measured parts of the array in follows, respectively. Minimization of the objective function over the mechanical field $\boldsymbol{x}$ yields

$$
\begin{gathered}
\left(\begin{array}{c}
\tilde{\boldsymbol{u}}_{(j)} \\
\boldsymbol{p}_{(j)}
\end{array}\right)=\boldsymbol{x}_{(j)}=\left(\begin{array}{c}
\tilde{\boldsymbol{u}}_{(j)}^{k} \\
\left(\mathbf{R}^{U U}\right)^{-1}\left(\boldsymbol{S}^{U}-\mathbf{R}^{U M} \tilde{\boldsymbol{u}}_{(j)}^{k}\right)
\end{array}\right) \\
\mathbf{R}=\left(( \mathbf { D } ( \boldsymbol { \theta } _ { ( j - 1 ) } ) \mathbf { R } - c _ { 2 } c _ { 1 } \mathbf { A } ^ { + } \mathbf { M } , - \mathbf { Z } ) ^ { T } \mathbf { D } ( \boldsymbol { \theta } _ { ( j - 1 ) } ) ^ { - 1 } \left(\left(\mathbf{D}\left(\boldsymbol{\theta}_{(j-1)}\right) \mathbf{R}-c_{2} c_{1} \mathbf{A}^{+} \mathbf{M},-\mathbf{Z}\right)\right.\right. \\
\boldsymbol{S}=\left(\left(\mathbf{D}\left(\boldsymbol{\theta}_{(j-1)}\right) \mathbf{R}-c_{2} c_{1} \mathbf{A}^{+} \mathbf{M},-\mathbf{Z}\right)^{T} \mathbf{D}\left(\boldsymbol{\theta}_{(j-1)}\right)^{-1} c_{2} \mathbf{A}^{+} \boldsymbol{a} \sqrt{S_{0}(\omega)} .\right.
\end{gathered}
$$

As $\boldsymbol{p}_{(j)}$ is obtained, $\tilde{\boldsymbol{\sigma}}_{(j)}$ could be easily assembled based on equation (15). 


\section{A.3 UMP step}

To update the damage parameter $\boldsymbol{\theta}$, the objective function in $\left(\mathrm{P}_{s}\right)$ is divided by each element $e$

$$
\begin{aligned}
e_{\mathrm{CRE}}^{e}\left(\tilde{\boldsymbol{u}}_{(j)}^{e}, \tilde{\boldsymbol{\sigma}}_{(j)}^{e}, \theta_{e}\right) & =\frac{1}{2} \int_{\omega_{\min }}^{\omega_{\max }}\left\|\left(\theta_{e} \mathbf{D}_{e}\right)^{-\frac{1}{2}}\left(\theta_{e} \mathbf{D}_{e} \mathbf{R}_{e} \tilde{\boldsymbol{u}}_{(j)}^{e}-\tilde{\boldsymbol{\sigma}}_{(j)}^{e}\right)\right\|^{2} z(\omega) d \omega+\lambda\left|\theta_{e}-\theta_{0 e}\right| \\
& =\left(a_{e} \theta_{e}+\frac{b_{e}}{\theta_{e}}+c_{e}\right)+\lambda\left|\theta_{e}-\theta_{0 e}\right|, e=1, \cdots, m \\
a_{e} & =\frac{1}{2} \int_{\omega_{\min }}^{\omega_{\max }} \tilde{\boldsymbol{u}}_{(j)}^{e T} \mathbf{R}_{e}^{T} \mathbf{D}_{e} \mathbf{R}_{e} \tilde{\boldsymbol{u}}_{(j)}^{e} z(\omega) d \omega, \\
b_{e} & =\frac{1}{2} \int_{\omega_{\min }}^{\omega_{\max }} \tilde{\boldsymbol{\sigma}}_{(j)}^{e T} \mathbf{D}_{e}^{-1} \tilde{\boldsymbol{\sigma}}_{(j)}^{e} z(\omega) d \omega, \\
c_{e} & : \text { constant term. }
\end{aligned}
$$

Minimization of the objective function over every single-variable $\theta_{e}$ in each element results in

$$
\begin{aligned}
\theta_{e(j)}= & \arg \min \left(a_{e} \theta_{e}+\frac{b_{e}}{\theta_{e}}+c_{e}\right)+\lambda\left|\theta_{e}-\theta_{0 e}\right| \\
= & \left\{\begin{array}{l}
\sqrt{\frac{b_{e}}{a_{e}+2 \lambda}}, \text { if } \lambda<\frac{1}{2}\left(\frac{b_{e}}{\theta_{0 e}^{2}}-a_{e}\right) \\
\sqrt{\frac{b_{e}}{a_{e}-2 \lambda}}, \text { else if } \lambda<\frac{1}{2}\left(a_{e}-\frac{b_{e}}{\theta_{0 e}^{2}}\right), e=1, \cdots, m . \\
\theta_{0 e}, \text { else } \lambda \geq \frac{1}{2}\left|a_{e}-\frac{b_{e}}{\theta_{0 e}^{2}}\right|
\end{array}\right.
\end{aligned}
$$

\section{References}

[1] Swagato Das, P Saha, and SK Patro. Vibration-based damage detection techniques used for health monitoring of structures: a review. Journal of Civil Structural Health Monitoring, 6 (3):477-507, 2016.

[2] Zhong-Rong Lu and Li Wang. An enhanced response sensitivity approach for structural damage identification: convergence and performance. International Journal for Numerical Methods in Engineering, 111(13):1231-1251, 2017.

[3] James MW Brownjohn, Alessandro De Stefano, You-Lin Xu, Helmut Wenzel, and A Emin Aktan. Vibration-based monitoring of civil infrastructure: challenges and successes. Journal of Civil Structural Health Monitoring, 1(3-4):79-95, 2011.

[4] Hamed Ebrahimian, Rodrigo Astroza, Joel P Conte, and Raymond A de Callafon. Nonlinear finite element model updating for damage identification of civil structures using batch bayesian estimation. Mechanical Systems and Signal Processing, 84:194-222, 2017.

[5] Guilherme Ferreira Gomes, Yohan Ali Diaz Mendez, Patrícia da Silva Lopes Alexandrino, Sebastiao Simões da Cunha, and Antonio Carlos Ancelotti. A review of vibration based 
inverse methods for damage detection and identification in mechanical structures using optimization algorithms and ANN. Archives of Computational Methods in Engineering, pages $1-15,2018$.

[6] Stéphane Avril, Marc Bonnet, Anne-Sophie Bretelle, Michel Grédiac, François Hild, Patrick Ienny, Félix Latourte, Didier Lemosse, Stéphane Pagano, Emmanuel Pagnacco, et al. Overview of identification methods of mechanical parameters based on full-field measurements. Experimental Mechanics, 48(4):381, 2008.

[7] Giuseppe Geymonat and Stéphane Pagano. Identification of mechanical properties by displacement field measurement: a variational approach. Meccanica, 38(5):535-545, 2003.

[8] Felix Latourte, Andre Chrysochoos, Stwphane Pagano, and Bertrand Wattrisse. Elastoplastic behavior identification for heterogeneous loadings and materials. Experimental Mechanics, 48(4):435-449, 2008.

[9] Felix Latourte, André Chrysochoos, Bruno Berthel, André Galtier, Stéphane Pagano, and Bertrand Wattrisse. Local energy analysis of hcf fatigue using DIC \& IRT. In SEM 2009, pages Cd-Rom, 2009.

[10] Shaojuan Huang, Pierre Feissel, and Pierre Villon. Modified constitutive relation error: An identification framework dealing with the reliability of information. Computer Methods in Applied Mechanics and Engineering, 311:1-17, 2016.

[11] Mouldi Ben Azzouna, Pierre Feissel, and Pierre Villon. Robust identification of elastic properties using the modified constitutive relation error. Computer Methods in Applied Mechanics and Engineering, 295:196-218, 2015.

[12] Eric Florentin and Gilles Lubineau. Identification of the parameters of an elastic material model using the constitutive equation gap method. Computational Mechanics, 46(4):521$531,2010$.

[13] Jia Guo, Li Wang, and Izuru Takewaki. Modal-based structural damage identification by minimum constitutive relation error and sparse regularization. Structural Control and Health Monitoring, 25(12):e2255, 2018.

[14] Jia Guo, Li Wang, and Izuru Takewaki. Frequency response-based damage identification in frames by minimum constitutive relation error and sparse regularization. Journal of Sound and Vibration, 443:270-292, 2019. 
[15] Armen Der Kiureghian. A response spectrum method for random vibration analysis of mdf systems. Earthquake Engineering \& Structural Dynamics, 9(5):419-435, 1981.

[16] Maria-Giovanna Masciotta, Luís F Ramos, Paulo B Lourenço, Marcello Vasta, and Guido De Roeck. A spectrum-driven damage identification technique: Application and validation through the numerical simulation of the z24 bridge. Mechanical Systems and Signal Processing, 70:578-600, 2016.

[17] ZD Zheng, ZR Lu, WH Chen, and JK Liu. Structural damage identification based on power spectral density sensitivity analysis of dynamic responses. Computers $\&$ Structures, 146 : $176-184,2015$.

[18] Jiahao Lin, Wenshou Zhang, and Jianjun Li. Structural responses to arbitrarily coherent stationary random excitations. Computers \& structures, 50(5):629-633, 1994.

[19] Jiahao Lin, Yan Zhao, and Yahui Zhang. Accurate and highly efficient algorithms for structural stationary/non-stationary random responses. Computer Methods in Applied Mechanics and Engineering, 191(1-2):103-111, 2001.

[20] Jiahao Lin, Wenshou Zhang, and FW Williams. Pseudo-excitation algorithm for nonstationary random seismic responses. Engineering Structures, 16(4):270-276, 1994.

[21] JP Conte and BF Peng. Fully nonstationary analytical earthquake ground-motion model. Journal of Engineering Mechanics, 123(1):15-24, 1997.

[22] GML Gladwell and H Ahmadian. Generic element matrices suitable for finite element model updating. Mechanical Systems and Signal Processing, 9(6):601-614, 1995.

[23] Li Wang and Zhong-Rong Lu. Sensitivity-free damage identification based on incomplete modal data, sparse regularization and alternating minimization approach. Mechanical Systems and Signal Processing, 120:43-68, 2019.

[24] Costas Papadimitriou and Dimitra-Christina Papadioti. Component mode synthesis techniques for finite element model updating. Computers \& structures, 126:15-28, 2013.

[25] Arnaud Deraemaeker, Pierre Ladevèze, and $\mathrm{Ph}$ Leconte. Reduced bases for model updating in structural dynamics based on constitutive relation error. Computer methods in applied mechanics and engineering, 191(21-22):2427-2444, 2002. 
[26] D Barthe, A Deraemaeker, P Ladevèze, and S Le Loch. Validation and updating of industrial models based on the constitutive relation error. AIAA journal, 42(7), 2004.

[27] Amir Beck. On the convergence of alternating minimization for convex programming with applications to iteratively reweighted least squares and decomposition schemes. SIAM Journal on Optimization, 25(1):185-209, 2015.

[28] Koushik Roy and Samit Ray-Chaudhuri. Fundamental mode shape and its derivatives in structural damage localization. Journal of Sound and Vibration, 332(21):5584-5593, 2013.

[29] ZR Lu and SS Law. Features of dynamic response sensitivity and its application in damage detection. Journal of Sound and Vibration, 303(1-2):305-329, 2007.

[30] Manolis N Chatzis, Eleni N Chatzi, and Andrew W Smyth. An experimental validation of time domain system identification methods with fusion of heterogeneous data. Earthquake Engineering \& Structural Dynamics, 44(4):523-547, 2015.

[31] Eleni N Chatzi and Andrew W Smyth. The unscented Kalman filter and particle filter methods for nonlinear structural system identification with non-collocated heterogeneous sensing. Structural Control and Health Monitoring, 16(1):99-123, 2009.

[32] Eric M Hernandez. Identification of isolated structural damage from incomplete spectrum information using 11-norm minimization. Mechanical Systems and Signal Processing, 46(1): $59-69,2014$.

[33] Peter Van Overschee and Bart De Moor. N4sid: Subspace algorithms for the identification of combined deterministic-stochastic systems. Automatica, 30(1):75-93, 1994.

[34] Peter Van Overschee and BL De Moor. Subspace identification for linear systems: Theory-Implementation-Applications. Springer Science \& Business Media, 2012.

[35] John E Mottershead, Michael Link, and Michael I Friswell. The sensitivity method in finite element model updating: a tutorial. Mechanical systems and signal processing, 25(7): $2275-2296,2011$. 